\title{
Chronic cerebrospinal venous insufficiency and the doubtful promise of an endovascular treatment for multiple sclerosis
}

\author{
Howard Dorne, ${ }^{1}$ Osama 0 Zaidat, ${ }^{2}$ David Fiorella, ${ }^{3}$ \\ Joshua Hirsch, ${ }^{4}$ Charles Prestigiacomo, ${ }^{5}$ \\ Felipe Albuquerque, ${ }^{6}$ Robert W Tarr ${ }^{7}$
}

\section{INTRODUCTION}

Recently, a radically different concept regarding the pathogenesis of multiple sclerosis (MS) has been proposed. Termed chronic cerebrospinal venous insufficiency (CCSVI), it suggests that macro occlusive abnormalities of the extracranial venous drainage pathways of the brain and spinal cord can cause or contribute to MS. As a consequence of this theory, it has been suggested that angioplasty and possibly stenting of the internal jugular and/or azygos veins can improve the signs and symptoms of MS. These interventions have been performed sporadically across the globe in an open label fashion and never in the context of a well designed, controlled, randomized and blinded clinical trial. Despite this, the procedure has been labeled by some as 'liberation procedure' and caused a firestorm of interest in the medical and MS communities, both for and against its utilization. The arguments on all sides are passionate, ranging from the belief that venous intervention is a miracle cure that must not be withheld from patients, to the feeling that the procedure is ineffective and unwarranted at best and dangerous at worst. The various camps commonly protest that those with differing views are not acting in the best interest of their patients.

\footnotetext{
${ }^{1}$ St Joseph Hospital Health System, Orange, California, USA; ${ }^{2}$ Department of Neurology, Neurosurgery and Radiology, Froedtert Hospital/Medical College of Wisconsin, Milwaukee, Wisconsin, USA; ${ }^{3}$ Stony Brook University Medical Center, Stony Brook, New York, USA ${ }^{4}$ Massachusetts General Hospital, Boston, Massachusetts, USA; ${ }^{5}$ New Jersey Medical School, University of Medicine and Dentistry of New Jersey, Newark, New Jersey, USA; ${ }^{6}$ Barrow Neurological Institute, Phoenix, Arizona, USA; ${ }^{7}$ University Hospitals Case Medical Center, Cleveland, Ohio, USA
}

Correspondence to Dr R W Tarr, University Hospitals Case Medical Center, Cleveland, OH 44102, USA; robert.tarr@uhhospitals.org
As neurointerventionalists interested in interventional treatment of neurological disorders, it is time to take a thorough and objective look at CCSVI. This commentary will examine the origin of the CCSVI theory and discuss the data supporting and refuting its existence. An attempt will be made to critically analyze the available data and provide constructive recommendations about whether or not endovascular therapy represents a reasonable option at this point in time for patients with MS.

\section{BRIEF REVIEW OF MULTIPLE SCLEROSIS}

MS is a fearful and unpredictable disease that brings an enormous physical, emotional and financial burden on patients, family, relatives, friends and society in general. It is the most common cause of physical disability, with estimated 250000-350000 individual diagnosed with MS in the USA. The peak age at onset is $20-40$ years. It affects women more so than men and is more common among Caucasians. MS can present with just about any neurological symptom in any part of the nervous system, sensory, motor, cranial nerves, visual, autonomic, coordination and myelopathic on different occasions with cumulative disability. ${ }^{1}$ Diagnosis is based on clinical and imaging criteria (McDonald criteria) to establish the dissemination in place (different CNS sites) and time (at least 30 days between clinical relapses and 90 days for new MRI lesion without clinical relapse). The clinical course of $\mathrm{MS}$ is most commonly relapsing remitting, with return to baseline after each relapse, followed by secondary progressive starting as relapsing remitting, then primary progressive $\mathrm{MS}^{1}{ }^{1}$ The most prevalent hypothesis regarding the pathophysiological basis for MS is that it is an autoimmune inflammatory disease triggered by environmental factors (toxic and infectious triggers) with genetic predisposition leading to myelin and axonal destruction in the brain and spinal cord by the immune system. ${ }^{1}$ To date, MS management has been limited to the indefinite administration of 'disease modifying' medications and immune modulating agents which may reduce the number and severity of relapses. ${ }^{1}$ These agents are not only costly but are associated with a wide spectrum of side effects ranging from mild to severe.

\section{THE CCSVI THEORY AND SUPPORTIVE DATA}

In 2006, Zamboni, an Italian vascular surgeon, in an article titled 'The big idea: iron-dependent inflammation in venous disease and proposed parallels in multiple sclerosis' suggested that there were similarities between chronic venous disease of the extremities and $\mathrm{MS}^{2}$ He raised the possibility that venous reflux or obstruction in cerebral and spinal veins might have a relationship to $\mathrm{MS}{ }^{2}{ }^{2}$

Several years later, Zamboni et al reported on blinded transcranial and extracranial color Doppler sonographic findings in patients with MS and matched healthy controls and those with other neurological disorders. ${ }^{3}$ They focused on five findings: (1) reflux in the internal jugular vein (IJV) or vertebral veins $>0.88 \mathrm{~s}$; (2) reflux propagated in at least one out of the three deep cerebral veins $>0.55 \mathrm{~s}$; (3) high resolution $\mathrm{B}$ mode evidence of proximal IJV stenosis; (4) flow not Doppler detectable in the IJV or vertebral veins despite deep inspirations; and (5) negative difference of the cross sectional area of the IJV comparing the value obtained in the supine versus the sitting position. The authors concluded that detection of two or more of these findings constitutes the diagnosis of CCSVI. They found CCSVI in all MS patients and in none of the controls. The sensitivity, specificity, positive predictive value and negative predictive value of the test were all $100 \%$. They concluded that there was CCSVI in MS patients. ${ }^{3}$

In a second paper, Zamboni et al published that catheter venography in patients who met CCSVI Doppler criteria showed stenosis in the azygos vein $86 \%$ of the time and one or both IJV were affected in $91 \%$. In this study, the venographer was not blinded to the patients' diagnosis. ${ }^{4}$ The study proposed four venographic patterns: (A) large IJV with one IJV or proximal azygous vein stenosis; (B) both 
IJV and proximal azygos vein stenosis; (C) both IJV and normal azygous system; and (D) multilevel azygous stenosis with or without IJV involvement.

Finally, in 2009, Zamboni et al reported their results on the endovascular treatment of $65 \mathrm{MS}$ patients with CCSVI. ${ }^{5} \mathrm{No}$ isolated venous lesion was found, and the distribution of venographic patterns was $30 \%, 38 \%, 14 \%$ and $18 \%$ of types A to D, respectively. ${ }^{5}$ They performed percutaneous transluminal angioplasty (PTA) on all but one azygos lesion that did not respond to PTA alone and required stent placement. Pretreatment pressures beyond the stenosis were not significantly different than normal venous pressure and there was no significant change in pressure after angioplasty. Mean follow-up using extracranial Doppler was 18 months, with an overall restenosis rate of $47 \%$; more common in the jugular than azygos veins. Clinical outcome at 18 months was reported as showing relapse free of $50 \%$ versus $27 \%$ preoperatively. It is important to note that the interpretation of the clinical results of this uncontrolled study is confounded since patients were continued on 'immunomodulating' therapy after endovascular therapy. These medical therapies have been shown to significantly reduce relapse rates as well as the accumulation of MRI detectable enhancing lesions ${ }^{1}$ Finally, there was no improvement in patients with primary progressive or secondary progressive MS. ${ }^{5}$

\section{DATA AGAINST CCSVI ROLE IN MS}

Although the Zamboni papers have been quite supportive of CCSVI, there are a growing number of papers that raise serious questions about its validity. In early 2010, Khan et al described a number of independently accepted characteristics of venous disease and MS that contradict the CCSVI theory. ${ }^{6}$

1. Similar to other autoimmune diseases, $\mathrm{MS}$ is more common in young women while chronic venous insufficiency syndromes are not.

2. There are well known strong epidemiological associations between MS and geography, ethnicity, sun exposure, low vitamin $\mathrm{D}$ levels, gender, genetics and immigration studies that are not mirrored by chronic venous insufficiency.

3. Central veno-occlusive disease can lead to syndromes of idiopathic intracranial hypertension, ischemic and hemorrhagic infarcts and edema, none of which is typically seen in MS patients.
4. Vascular abnormalities related to chronically diminished venous flow would be expected to increase over time, yet after the age of 50 years the incidence of MS is quite low.

5. There is no other model of decreased venous drainage and an organ specific immune response.

6. Transient global ischemia is known to occur with jugular insufficiency but this entity is not seen in MS.

7. Radical neck dissections remove all jugular veins but they have never been seen to cause MS. ${ }^{6}$

The above cited challenges to the Zamboni thesis are based on largely theoretical considerations. In an attempt to replicate the Doppler findings of Zamboni, Doepp et al studied 56 MS patients and 20 controls using similar CCSVI criteria. ${ }^{7}$ The authors found no patients in either the MS or control groups who had the two or more criteria required for a diagnosis of CCSVI. They concluded based on these results as well as their extensive longitudinal experience with cranial venous Doppler ultrasound, that there is typically tremendous reserve capacity of the extrajugular pathways for cerebral venous drainage and that it is highly unlikely that IVJ stenosis would cause central venous congestion. Furthermore, they went on to discourage interventional procedures for CCSVI outside of the context of appropriately designed clinical research studies. ${ }^{8}$

Additionally, Sundstrom et al looked at MRI of 21 patients with relapsing remitting MS and 20 healthy controls, and found no differences in internal jugular venous outflow between the two groups. ${ }^{9}$ Finally, preliminary data from Zivadinov et al, from the MS research group at the State University of New York in Buffalo, presented findings in the first 500 participants studied with venous Doppler looking at the prevalence of CCSVI in MS patients and controls. Using the requirement that $\geq 2$ CCSVI Doppler criteria be met, CCSVI was found in $62.5 \%$ of $\mathrm{MS}$ patients, $25.9 \%$ of healthy controls and $45 \%$ of other neurological disorders. ${ }^{10}$ At least preliminarily, these results are different from the $100 \%$ sensitivity and specificity found by Zamboni and colleagues. ${ }^{3}$

\section{COMMENTARY}

There is little debate as to the potential ravages of $\mathrm{MS}$ and the sincere desire to improve outcomes in patients suffering from this terrible disease. As such, when seemingly miraculous cures are proffered, we believe that it is our responsibility as neurointerventionalists to rationally review its use.

There are few data to support the validity of CCSVI. The lack of data seems counterbalanced by the great hope for the miracle of an endovascular treatment for MS. The topic has caused widespread attention and debate in the media, medical literature and the internet. ${ }^{11-17}$ As of late August 2010, a Google search on 'liberation procedure' yielded about 2650000 results and approximately 181000 for 'CCSVI'. Sponsored links appear for treatment in Mexico, Poland, Costa Rica, India and other locations. At least one toll free telephone number akin to '1-800-I Treat MS' has been created. ${ }^{18}$

The prospect of opening an open label, non-study related MS endovascular CCSVI practice can be very seductive. For physicians, the barriers to entry are small since most interventionalists are technically able to perform these procedures and the required devices are readily available. At the same time, there are many patients who are desperate for a procedure which might improve their condition despite the lack of evidence to support its benefits and almost regardless of its potential risks. Indeed, some might argue that because the procedure is safe, if there is any possibility of ameliorating some of the symptoms of MS patients the procedure should be offered to them. However, no invasive procedure is completely safe. In fact, there are increasing reports of complications related to PTA or stenting for CCSVI, including intracranial hemorrhage, stent migration into the heart and jugular vein thrombosis ${ }^{19}$

The moniker, 'liberation procedure', is a marketer's dream and by itself suggests unrealistic but compelling expectations. Many patients are willing to pay cash, sometimes tens of thousands of dollars, for a single procedure. Many patients rave about their procedures, yet outside of a well controlled trial, it is hard to disprove the placebo effect and prove the true clinical benefits.

In view of the forgoing, and in an attempt to help resolve the CCSVI conundrum, it would seem that the fundamental questions are:

1. Is there a cause and effect relationship between CCSVI and MS, and in which direction does this work?

2. If CCSVI does cause or worsen MS, should this be treated with endovascular therapies?

3. If endovascular treatment is contemplated, which therapy should be offered 
and under what technical and clinical circumstances should they be applied?

There is paramount need for credible scientific evidence that will allow us to address these questions. Firstly, we should encourage trials using non-invasive studies to test if CCSVI-MS actually exists. At the current time, the corroboratory evidence supporting Zamboni's initial findings of an association between CCSVI and $\mathrm{MS}$ are limited. In fact, the majority of additional evidence-including the work of Doepp et al and Sundstrom et al, cited in this review-actually failed to replicate the findings of Zamboni and colleagues. ${ }^{7-9}$

Moreover, the early results of Zivadinov et al are also not very compelling. ${ }^{10}$ In addition, the initial claim by Zamboni et al that they had developed a perfect test for CCSVI-MS raises serious questions about the credibility of their evidence. As pointed out by Novella, few if any tests in medicine have $100 \%$ sensitivity and $100 \%$ specificity. $^{20}$

Fortunately, the US and Canadian MS societies have undertaken seven studies to investigate the CCSVI-MS association. ${ }^{14}{ }^{15}$ The necessity of requiring an invasive diagnostic study such as catheter venography to evaluate the CCSVI-MS association is more difficult to reconcile at this point, particularly since the seminal findings of Zamboni et al which initiated this entire controversy were based on noninvasive Doppler ultrasound.

If the association between CCSVI and MS cannot be confirmed, then further studies evaluating CCSVI treatment are unnecessary. While it could be argued that even if the prevalence of venous 'abnormalities' is similar in patients with MS and controls, venous intervention in MS should still be studied since MS patients might be more susceptible to the detrimental effects of CCSVI than normal patients, this position seems tenuous at best.

If an association between CCSVI and MS can be established, then the next logical step would be to design multicenter randomized clinical trials to assess the benefits of endovascular interventions.

\section{CONCLUSION}

More evidence is needed to establish the association between CCSVI and MS. If more solid clinical evidence can confirm that the CCSVI-MS relationship is real, randomized clinical trials will be required to assess the benefits of endovascular interventions. If these trials establish a benefit for endovascular therapy, then at that point treatment can be made widely available. However, until these steps are taken, in our opinion, there is no role for the endovascular treatment of CCSVI in the MS patient outside of approved clinical trials.

\section{Competing interests None.}

Provenance and peer review Not commissioned; not externally peer reviewed.

Accepted 29 September 2010

Published Online First 23 October 2010

J Neurolntervent Surg 2010;2:309-311.

doi:10.1136/pnis.2010.003947

\section{REFERENCES}

1. Compston A, Coles A. M1 "Multiple sclerosis". Lancet 2008;372:1502-17.

2. Zamboni P. The big idea: iron-dependent inflammation in venous disease and proposed parallels in multiple sclerosis. J R Soc Med 2006;99:589-93.

3. Zamboni $\mathbf{P}$, Menegatti $E$, Galeotti $R$, et al. The value of cerebral Doppler venous haemodynamics in the assessment of multiple sclerosis. J Neurol Sci 2009:282:21-7.

4. Zamboni P, Galeotti R, Menegatti E, et al. Chronic cerebrospinal venous insufficiency in patients with multiple sclerosis. J Neurol Neurosurg Psychiatry 2009:80:392-9

5. Zamboni P, Galeotti R, Menegatti E, et al. A prospective open-label study of endovascular treatment of chronic cerebrospinal venous insufficiency. J Vasc Surg 2009;50:1348-58.e1-3.

6. Khan 0, Filippi M, Freedman MS, et al. Chronic cerebrospinal venous insufficiency and multiple sclerosis. Ann Neurol 2010;67:286-90.
7. Doepp F, Paul F, Valdueza J, et al. No cerebrocervical venous congestion in patients with multiple sclerosis. Ann Neurol 2010;68:173-83.

8. Doepp F, Schreiber SJ, von Munster T, et al. How does the blood leave the brain? A systematic ultrasound analysis of cerebral venous drainage patterns. Neuroradiology 2004;46:565-70

9. Sundström P, Wåhlin A, Ambarki K, et al. Venous and cerebrospinal fluid flow in multiple sclerosis: a casecontrol study. Ann Neurol 2010;68:255-9.

10. Zivadinov R, Marr K, Ramanathan $\mathrm{M}$, et al. Combined Transcranial and Extracranial Venous Doppler Evaluation (CTEVD Study). Description of the design and interim results of an epidemiological study of the prevalence of chronic cerebrospinal venous insufficiency in MS and related diseases. Neurology 2010;74(P06.144):A545.

11. Burton TM. "MS program halted amid controversy". Wall Str J 2010. http://online.wsj.com/article/ SB100014240527487042117045751403139043352 40.html?mod=wsj_hpp_MIDDLTopStories (accessed 14 Aug 2010)

12. Grady D. "From MS patients, outcry for unproved treatment". New York Times 2010. http://www. nytimes.com/2010/06/29/health/29vein.htm (accessed 14 Aug 2010).

13. Burton TM. "Studies cast doubt on new MS theory". Wall Street J 2010. http://online.wsj.com/article/ SB100014240527487037879045754031601557103 80.html (accessed 14 Aug 2010).

14. Multiple Sclerosis Society of Canada. CCSVI and MS: overview and FAO. Multiple sclerosis society of Canada, 2010. http://mssociety.ca/en/research/ medmmo_20091021_faq.html. (accessed 14 Aug 2010).

15. National Multiple Sclerosis Society. Update CCSVI: pursuing promising avenues in MS research. National Multiple Sclerosis Society, 2010. http:// www.nationalmssociety.org/news/news-detail/index aspx?nid=2206 (accessed 14 Aug 2010)

16. Stanbrook MB, Hébert PC. Access to treatment for multiple sclerosis must be based on science, not hope. CMAJ 2010;182:1151.

17. News-Medical.Net. Research shows no link among CCSVI and development of multiple sclerosis. NewsMedical.Net, 2010. http://www.news-medical.net/ news/20100802/Research-shows-no-link-betweenCCSVI-and-development-of-multiple-sclerosis.aspx (accessed 14 Aug 2010).

18. http://www.pacificinterventional.com/about_ccsvi. html. (accessed 14 Aug 2010).

19. Sclafani SJA. Chronic cerebrospinal insufficiency syndrome: new paradigm and therapy for multiple sclerosis. I Endovascular Today 2010:41-6.

20. Novella, Steven. 2010. CCSVI-The importance of replication. NeuroLogica Blog. http://www.theness com/neurologicablog/?p=2172 (accessed Aug 14). 
5. Strunk HM, Schild H. Actual clinical use of gadolinium-chelates for non-MRI applications. Eur Radiol 2004;14:1055-62.

6. Nyman U, Elmstahl B, Leander P, et al. Are gadolinium-based contrast media really safer than iodinated media for digital subtraction angiography in patients with azotemia? Radiology 2002;223:311-18.

7. Heinrich MC, Kuhlmann MK, Kohlbacher S, et al. Cytotoxicity of iodinated and gadolinium-based contrast agents in renal tubular cells at angiographic concentrations: in vitro study. Radiology 2007;242:425-34.

8. Thompson HS, Almen T, Morcos SK. Gadolinium-containing contrast media for radiographic examinations: a position paper. Eur Radiol 2002;12:2600-5.

9. Remy-Jardin M, Dequiedt P, Ertzbischoff 0, et al. Safety and effectiveness of gadolinium-enhanced multidetector row spiral CT angiography of the chest: preliminary results in 37 patients with contraindications to iodinated contrast agents. Radiology 2005;235:819-26.
10. Kuo P, Kanal E, Abu-Alfa AK, et al. Contrast agents and nephrogenic systemic fibrosis. Radiology 2007;242:647-9.

11. Rudin S, Yang CY, Yang Z, et al. The clinical potential of $\mathrm{x}$-ray flat panel detectors for dynamic - evaluation of animal angiographic studies using a direct conversion-type flat panel detector. Toshiba Med Rev 2000;73:1-12.

12. Arsenault TM, King BF, Marsh JW Jr, et al. Systemic gadolinium toxicity in patients with renal insufficiency and renal failure: retrospective analysis of an initial experience. Mayo Clin Proc 1996;71:1150-4.

13. Sancak T, Bigic S, Sanlidilek U. Gadodiamide as an alternative contrast agent in intravenous digital subtraction angiography and interventional procedures of the upper extremity veins. Cardiovasc Intervent Radiol 2002;25:49-52.

14. Prince MR, Arnoldus C, Frisoli JK. Nephrotoxicity of high-dose gadolinium compared with iodinated contrast. J Magn Reson Imaging 1996;6:162-6.

15. Thomsen HS. Gadolinium-based contrast media may be nephrotoxic even at approved doses. Eur Radiol 2004;14:1654-6.

\section{Corrections}

There was an error in an article published in the December 2010 issue of the journal (Chronic cerebrospinal venous insufficiency and the doubtful promise of an endovascular treatment for multiple sclerosis. J NeuroIntervent Surg 2010;2:309-11. Published Online First: 23 October 2010. doi:10.1136/jnis.2010.003947). On page 310, second paragraph, fourth sentence should read: 'Pretreatment pressures beyond the stenosis were not significantly different from normal venous pressure, however, a change in pressure was demonstrated after angioplasty'.

J Neurolntervent Surg 2011;3:97. doi:10.1136/jnis.2010.003947corr1

Several author names were omitted from an article published in the December 2010 issue of the journal (The POST trial: initial post-market experience of the Penumbra system: revascularization of large vessel occlusion in acute ischemic stroke in the United States and Europe. J NeuroIntervent Surg 2010;2:341-4. doi:10.1136/jnis.2010.002600). The correct author list is as follows:

Authors: Robert Tarr ${ }^{1}$, Dan $\mathrm{Hsu}^{1}$, Zsolt Kulcsar ${ }^{2}$, Christophe Bonvin ${ }^{2}$, Daniel Rufenacht ${ }^{2}$, Karsten Alfke ${ }^{3}$, Robert Stingele ${ }^{3}$, Olav Jansen ${ }^{3}$, Donald Frei ${ }^{4}$, Richard Bellon ${ }^{4}$, Michael Madison $^{5}$, Tobias Struffert ${ }^{6}$, Arnd Dorfler ${ }^{6}$, Iris Q. Grunwald ${ }^{7}$, Wolfgang Reith ${ }^{7}$, Anton Haass ${ }^{7}$.

Affiliations: ${ }^{1}$ University Hospital, Cleveland, $\mathrm{OH},{ }^{2}$ Hôpitaux Universitaires de Genève, Geneve, Switzerland, ${ }^{3}$ University of Kiel Medical Center, Kiel, Germany, ${ }^{4}$ Swedish Med. Ctr., Denver, CO, ${ }^{5}$ St. Paul's United Hosp., St. Paul, MN, ${ }^{6}$ Universitatsklinikum Erlangen, Germany, ${ }^{7}$ Universitätsklinikum des Saarlandes, Germany.

Robert Tarr (Robert.Tarr@UHhospitals.org)

Dan Hsu (Dan.Hsu@UHhospitals.org)

Zsolt Kulscar (kulcsarzsolt22@gmail.com)

Christophe.Bonvin (Christophe.Bonvin@hcuge.ch)

Daniel Rufenacht (daniel.rufenacht@hirslanden.ch)

Karsten Alfke (k.alfke@neurorad.uni-kiel.de)

Robert Stingele (r.stingele@neurologie.uni-kiel.de)

Olav Jansen (o.jansen@neurorad.uni-kiel.de)

Donald Frei (don.frei@riaco.com)

Richard Bellon (Richard.Bellon@riaco.com)

Michael Madison (mmadison@stpaulrad.com)

Tobias Struffert (tobias.struffert@nrad.imed.uni-erlangen.de)

Arnd Dorfler (a.doerfler@nrad.imed.uni-erlangen.de)

Iris Q. Grunwald (I.grunwald@gmx.net)

Wolfgang Reith (nrreith@uniklinikum-saarland.de)

Anton Haass (neahaa@uniklinik-saarland.de)

J Neurolntervent Surg 2011;3:97. doi:10.1136/jnis.2010.002600corr1 